\title{
Some Historical Comments about Interface Research
}

\author{
M. Rühle
}

MPI for Metals Research, Heisenbergstr. 3, 70569 Stuttgart, Germany

Ewing and Rosenhain [1] suggested that "metal grains are, in fact, crystals, except that each of their bounding surfaces is casually determined by the meeting of one grain with another". Several suggestions were put forward concerning the structure (crystallinity) of grain boundaries. The community accepted in those early days that the grains of a polycrystalline material were "glued together by a thin amorphous layer" ("amorphous cement theory") which covers the grain surfaces.

Jeffries and Archer [2] suggested 3 alternative models for the grain boundary in a metal:

- there are voids between the two crystals,

- there is a zone in which some of the atoms are held in both crystal lattices, in which case the lattices would be distorted at the surface of the corresponding grains, - there is a zone of disorganised or amorphous metal.

Recently, O.B.M. Hardouin Duparc [3] pointed out that French scientist G. Friedel [4] proposed already in 1904 a geometrical description of the crystalline nature of specific grain boundaries ("special boundaries"). This "inverse density of coincident lattice site" was later "reinvented" by several scientists, see [3]. The results of theoretical and experimental studies are summarised by Murr [5] (until 1973) and Sutton and Balluffi [6] (until 1996). With the advent of new quantitative experimental techniques, such as X-ray scattering and high-resolution TEM, detailed information on special, mostly highly symmetrical grain boundaries were obtained.

In 1982 the very first meeting specialising on the structure of internal interfaces and boundaries was held in Caen/France (September 6 - 9), at the time called "Structure and Properties of Intergranular Boundaries" [7]. Many of the attendees of the 1982 meeting are still active in the field of interface science! The 1982 meeting resulted from a French-US collaborative program which included also scientists from other nations. The meeting was organised by S. Hagege, G. Nouet, G. Sainfort and S.L. Sass. Since then every 3 years an international meeting on Intergranular and Interface Boundaries in Materials (iib) is being held. The $13^{\text {th }}$ iib meeting was held in Shima/Mie, Japan, from June 27 to July 2, 2010.

Enormous progress had been made concerning interfaces in science and technology. This is true for theoretical studies as well as experimental investigations. Progress is marginal in some fields; in others it is very impressive. So far, theoretical as well as experimental studies on the atomistics of interfaces concentrate on highly symmetrical boundaries. Many very detailed results are reported for special boundaries, including small-angle grain boundaries. The technologically more important general interfaces are not so accessible to both, theoretical and experimental studies. This will be shown for grain boundaries in alumina and strontium titanate. In the future, new concepts, techniques and ideas have to be developed allowing tackling this difficult task. 


\section{References}

[1] J.A. Ewing and W. Rosenhain, Proc. Roy. Soc. A65 (1899) 85.

[2] Z. Jeffries and R.S. Archer, Science of Metals, McGraw-Hill (1924) p 73

[3] O.B.M. Hardouin Duparc, Journal of Materials Science, to be published (2011)

[4] G. Friedel "Études sur les groupements cristallins". Bulletin de la Société de l'Industrie Minérale 3:877-1097; 4:127-273; 4:479-592 (1904-1905).

[5] L.E. Murr, "Interfacial Phenomena in Metals and Alloys", Addison and-Wesley, Reading (1974).

[6] A.P. Sutton and RW. Balluffi, "Interfaces in Crystalline Materials", Oxford University Press, Oxford (1996)

[7] S. Hagege, G. Nouet, Journal de Physique C6 (1982). 